\title{
A Jump-Start for Astronomy Education in Taiwan
}

Wen-Ping Chen, Institute of Physics and Astrnomy, National Central University, Chung Li, Taiwan 32054, China $R$.

Spurred by the leaping developments of research activities (SMA, TAOS, AMIBA), Taiwan is catching up in virtually all aspects of its education in astronomy. The first astronomy research institute was established by the Academia Sinica about 10 years ago, which catalyzed within two years the first graduate school of astronomy, as well as an elaborate astronomy museum. Since then astronomy education at all levels, from colleges to primary schools, has been booming. More than a dozen universities are offering astronomy courses, and two more graduate schools will soon be instituted. Textbooks get written, and books on popular science, either translated or composed by local authors, have mushroomed on the market. I will outline these ongoing activities along with plans on the horizon.

\section{An Interactive Approach to Planetary Orbits at Secondary Level}

V.B.Bhatia, Department of Physics and Astronomy, University of Delhi, New Delhi 110 007, India

I describe an interactive unit for teaching/learning planetary orbits at secondary level. The unit consists of a pre-test, activites related to the planetary orbits and a post-test. The pre-test is designed to modulate the activities to suit the needs of the students. The activities are (1) Trajectory of a particle in a gravitational field when the field is constant, and when it varies with distance (invoking a fictitious planet WonderX); (2) Plotting the orbit of a planet round the sun starting from select initial conditions and discovering Kepler's laws; (3) Plotting orbits with force laws containing terms like $1 / r^{3}$ and $1 / r^{4}$ and discovering precession. Students work through graded steps. To help them, simple numerical algorithms and computer programs have been developed as they are not yet comfortable with calculus. A post-test gauges the progress of the students for a possible revisit to the topic. Such units can be very effective in teaching basic astronomy in developing countries.

\section{An Undergraduate Program for Astronomy in México}

Hector Bravo-Alfaro et al., Departamento de Astronomía, Universidad de Guanajuato, Guanajato GTO 36000, México

Astronomy in México has an ancient tradition, reinforced during the twentieth century by groups working in theoretical and observational astronomy. During the 1990s, the Great Millimeter Telescope (a single 50-m antenna) has been approved, and a 6-m infrared telescope is under study. Graduate and undergraduate programs must be improved to prepare future Mexican and Latin 